\title{
JONES POLYNOMIALS, VOLUME AND ESSENTIAL KNOT SURFACES: A SURVEY
}

\author{
DAVID FUTER, EFSTRATIA KALFAGIANNI, AND JESSICA S. PURCELL
}

\begin{abstract}
This paper is a brief overview of recent results by the authors relating colored Jones polynomials to geometric topology. The proofs of these results appear in the papers [18, 19, while this survey focuses on the main ideas and examples.
\end{abstract}

\section{INTRODUCTION}

To every knot in $S^{3}$ there corresponds a 3 -manifold, namely the knot complement. This 3 -manifold decomposes along tori into geometric pieces, where the most typical scenario is that all of $S^{3} \backslash K$ supports a complete hyperbolic metric [42]. Incompressible surfaces embedded in $S^{3} \backslash K$ play a crucial role in understanding its classical geometric and topological invariants.

The quantum knot invariants, including the Jones polynomial and its relatives, the colored Jones polynomials, have their roots in representation theory and physics [27, 45], and are well connected to topological quantum field theory [47]. While the constructions of these invariants seem to be unrelated to the geometries of 3-manifolds, in fact topological quantum field theory predicts that the Jones polynomial knot invariants are closely related to the hyperbolic geometry of knot complements [46. In particular, the volume conjecture of R. Kashaev, H. Murakami, and J. Murakami [28, 36, 35, 12] asserts that the volume of a hyperbolic knot is determined by certain asymptotics of colored Jones polynomials. There is also growing evidence indicating direct relations between the coefficients of the Jones and colored Jones polynomials and the volume of hyperbolic links. For example, numerical computations show such relations [6], as do theorems proved for several classes of links, including alternating links [10], closed 3-braids [16], highly twisted links [14], and certain sums of alternating tangles [15].

In a recent monograph [19, the authors have initiated a new approach to studying these relations, focusing on the topology of incompressible surfaces in knot complements. The motivation behind studying surfaces is as follows. On the one hand, certain spanning surfaces of knots have been shown to carry information on colored Jones polynomials [8]. On the other hand, incompressible surfaces also shed light on volumes of manifolds [2] and additional geometry and topology (e.g. [1, 33, 34]). With these ideas in mind, we developed

D.F. is supported in part by NSF grant DMS-1007221.

E.K. is supported in part by NSF grants DMS-0805942 and DMS-1105843.

J.P. is supported in part by NSF grant DMS-1007437 and a Sloan Research Fellowship.

October 15, 2018. 
a machine that allows us to establish relationships between colored Jones polynomials and topological/geometric invariants.

The purpose of this paper is to give an overview of recent results, especially those of [19], and some of their applications. The content is an expanded version of talks given by the authors at the conferences Topology and geometry in dimension three, in honor of William Jaco, at Oklahoma State University in June 2010; Knots in Poland III at the Banach Center in Warsaw, Poland, in July 2010; as well as in seminars and department colloquia. This paper includes background and motivation, along with several examples that did not appear in the original lectures. Many figures in this survey are drawn from slides for those lectures, as well as from the papers [19, 14, 18].

This paper is organized as follows. In sections 1, 2, and 3, we develop several connections between (colored) Jones polynomials and topological objects of the corresponding dimension. That is, Section 1 describes the connection between these polynomial invariants and certain state graphs associated to a link diagram. Section 2 describes the state surfaces associated to these state graphs, and explains the connection of these surfaces to the sequence of degrees of the colored Jones polynomial. Section 3 dives into the 3dimensional topology of the complement of each state surface, and contains most of our main theorems. In Section 4, we illustrate the main theorems with a detailed example. Finally, in Section 5, we describe the polyhedral decomposition that plays a key role in our proofs.

\section{State graphs and the Jones polynomial}

The first objects we consider are 1-dimensional: graphs built from the diagram of a knot or link. We will see that these graphs have relationships to the coefficients of the colored Jones polynomials, and that a ribbon version of one of these graphs encodes the entire Jones polynomial. In later sections, we will also see relationships between the graphs and quantities in geometric topology.

1.1. Graphs and state graphs. Associated to a diagram $D$ and a crossing $x$ of $D$ are two link diagrams, each with one fewer crossing than $D$. These are obtained by removing the crossing $x$, and reconnecting the diagram in one of two ways, called the $A$-resolution and $B$-resolution of the crossing, shown in Figure 1 .
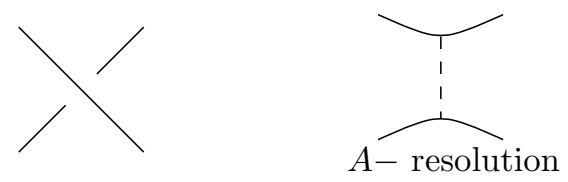

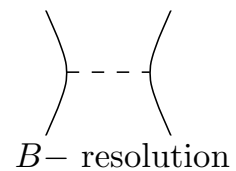

Figure 1. $A$ - and $B$-resolutions of a crossing.

For each crossing of $D$, we may make a choice of $A$-resolution or $B$-resolution, and end up with a crossing-free diagram. Such a choice of $A$ - or $B$-resolutions is called a Kauffman state, denoted $\sigma$. The resulting crossing-free diagram is denoted by $s_{\sigma}$.

The first graph associated with our diagram will be trivalent. We start with the crossingfree diagram given by a state. The components of this diagram are called state circles. For 

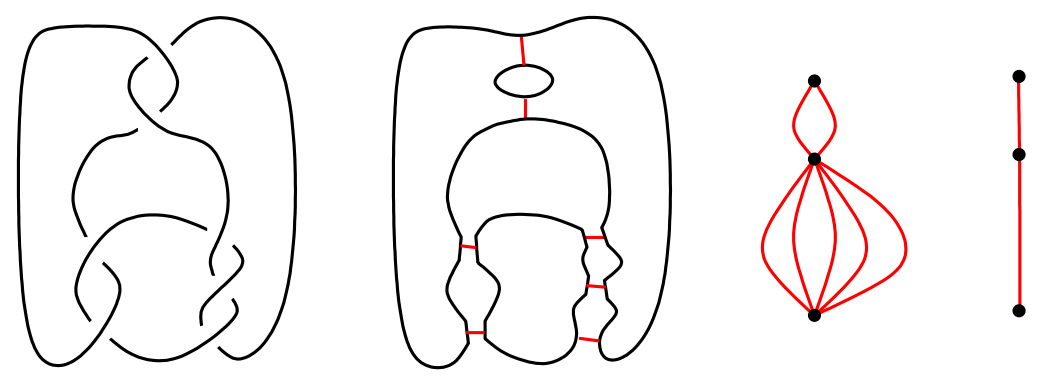

Figure 2. Left to right: A diagram, the graphs $H_{A}, \mathbb{G}_{A}$, and $\mathbb{G}_{A}^{\prime}$.

each crossing $x$ of $D$, attach an edge from the state circle on one side of the crossing to the other, as in the dashed lines of Figure 1. Denote the resulting graph by $H_{\sigma}$. Edges of $H_{\sigma}$ come from state circles and crossings; there are two trivalent vertices for each crossing.

To obtain the second graph, collapse each state circle of $H_{\sigma}$ to a vertex. Denote the result by $\mathbb{G}_{\sigma}$. The vertices of $\mathbb{G}_{\sigma}$ corespond to state circles, and the edges correspond to crossings of $D$. The graph $\mathbb{G}_{\sigma}$ is called the state graph associated to $\sigma$.

In the special case where each state circle of $\sigma$ traces a region of the diagram $D(K)$, the state graph $\mathbb{G}_{\sigma}$ is called a checkerboard graph or Tait graph. These checkerboard graphs record the adjacency pattern of regions of the diagram, and have been studied since the work of Tait and Listing in the 19th century. See e.g. [38, Page 264].

Our primary focus from here on will be on the all $-A$ state, which consists of choosing the $A$-resolution at each crossing, and similarly the all- $B$ state. Their corresponding state graphs are denoted $\mathbb{G}_{A}$ and $\mathbb{G}_{B}$. An example of a diagram, as well as the graphs $H_{A}$ and $\mathbb{G}_{A}$ that result from the all- $A$ state, is shown in Figure 2.

For the all- $A$ and all- $B$ states, we define graphs $\mathbb{G}_{A}^{\prime}$ and $\mathbb{G}_{B}^{\prime}$ by removing all duplicate edges between pairs of vertices of $\mathbb{G}_{A}$ and $\mathbb{G}_{B}$, respectively. Again see Figure 2 ,

The following definition, formulated by Lickorish and Thistlethwaite [31, 41], captures the class of link diagrams whose Jones polynomial invariants are especially well-behaved.

Definition 1.1. A link diagram $D(K)$ is called $A$-adequate (resp. $B$-adequate) if $\mathbb{G}_{A}$ (resp. $\mathbb{G}_{B}$ ) has no 1 -edge loops. If $D(K)$ is both $A$ and $B$-adequate, then $D(K)$ and $K$ are called adequate.

We will devote most of our attention to $A$-adequate knots and links. Because the mirror image of a $B$-adequate knot is $A$-adequate, this includes the $B$-adequate knots up to reflection. We remark that the class of $A$ - or $B$-adequate links is large. It includes all prime knots with up to 10 crossings, alternating links, positive and negative closed braids, closed 3-braids, Montesinos links, and planar cables of all the above [31, 40, 41]. In fact, Stoimenow has computed that there are only two knots of 11 crossings and a handful of 12 crossing knots that are not $A$ - or $B$-adequate. Furthermore, among the 253,293 prime knots with 15 crossings tabulated in Knotscape [24], at least 249,649 are either $A$-adequate or $B$-adequate [40]. 
Recall that a link diagram $D$ is called prime if any simple closed curve that meets the diagram transversely in two points bounds a region of the projection plane without any crossings. A prime knot or link admits a prime diagram.

1.2. The Jones polynomial from the state graph viewpoint. Here, we recall a topological construction that allows us to recover the Jones polynomial of any knot or link from a certain 2-dimensional embedding of $\mathbb{G}_{A}$.

A connected link diagram $D$ leads to the construction of a Turaev surface [44, as follows. Let $\Gamma \subset S^{2}$ be the planar, 4 -valent graph of the link diagram. Thicken the (compactified) projection plane to a slab $S^{2} \times[-1,1]$, so that $\Gamma$ lies in $S^{2} \times\{0\}$. Outside a neighborhood of the vertices (crossings), our surface will intersect this slab in $\Gamma \times[-1,1]$. In the neighborhood of each vertex, we insert a saddle, positioned so that the boundary circles on $S^{2} \times\{1\}$ are the components of the $A$-resolution $s_{A}(D)$, and the boundary circles on $S^{2} \times\{-1\}$ are the components of $s_{B}(D)$. (See Figure 3.) Then, we cap off each circle with a disk, obtaining an unknotted closed surface $F(D)$.

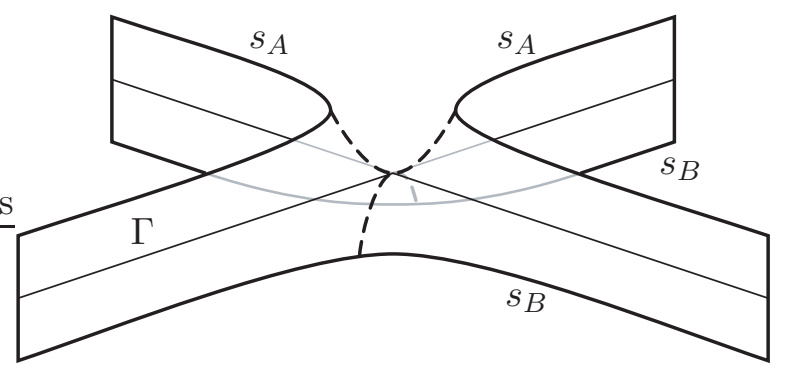

FiguRE 3. Near each crossing of the diagram, a saddle surface interpolates between circles of $s_{A}(D)$ and circles of $s_{B}(D)$. The edges of $\mathbb{G}_{A}$ and $\mathbb{G}_{B}$ can be seen as gradient lines at the saddle.

In the special case when $D$ is an alternating diagram, each circle of $s_{A}(D)$ or $s_{B}(D)$ follows the boundary of a region in the projection plane. Thus, for alternating diagrams, the surface $F(D)$ is exactly the projection sphere $S^{2}$. For general diagrams, it is still the case that the knot or link has an alternating projection to $F(D)$ [8, Lemma 4.4].

The construction of the Turaev surface $F(D)$ endows it with a natural cellulation, whose 1 -skeleton is the graph $\Gamma$ and whose 2-cells correspond to circles of $s_{A}(D)$ or $s_{B}(D)$, hence to vertices of $\mathbb{G}_{A}$ or $\mathbb{G}_{B}$. These 2 -cells admit a natural checkerboard coloring, in which the regions corresponding to the vertices of $\mathbb{G}_{A}$ are white and the regions corresponding to $\mathbb{G}_{B}$ are shaded. The graph $\mathbb{G}_{A}$ (resp. $\mathbb{G}_{B}$ ) can be embedded in $F(D)$ as the adjacency graph of white (resp. shaded) regions. Note that the faces of $\mathbb{G}_{A}$ (that is, regions in the complement of $\mathbb{G}_{A}$ ) correspond to vertices of $\mathbb{G}_{B}$, and vice versa. In other words, the graphs are dual to one another on $F(D)$.

A graph, together with an embedding into an orientable surface, is often called a ribbon graph. Ribbon graphs and their polynomial invariants have been studied by many authors, including Bollobas and Riordan [4, 5]. Building on this point of view, Dasbach, Futer, Kalfagianni, Lin and Stoltzfus [8] showed that the ribbon graph embedding of $\mathbb{G}_{A}$ into the 
Turaev surface $F(D)$ carries at least as much information as the Jones polynomial $J_{K}(t)$. To state the relevant result from [8], we need the following definition.

Definition 1.2. A spanning subgraph of $\mathbb{G}_{A}$ is a subgraph that contains all the vertices of $\mathbb{G}_{A}$. Given a spanning subgraph $\mathbb{G}$ of $\mathbb{G}_{A}$ we will use $v(\mathbb{G}), e(\mathbb{G})$ and $f(\mathbb{G})$ to denote the number of vertices, edges and faces of $\mathbb{G}$ respectively.

Theorem $1.3([8])$. Let $D$ be a connected link diagram. Then the Kauffman bracket $\langle D\rangle \in \mathbb{Z}\left[A, A^{-1}\right]$ can be expressed as

$$
\langle D\rangle=\sum_{\mathbb{G} \subset \mathbb{G}_{A}} A^{e\left(\mathbb{G}_{A}\right)-2 e(\mathbb{G})}\left(-A^{2}-A^{-2}\right)^{f(\mathbb{G})-1},
$$

where $\mathbb{G}$ ranges over all the spanning subgraphs of $\mathbb{G}_{A}$.

Recall that given a diagram $D$, the Jones polynomial $J_{K}(t)$ is obtained from the Kauffman bracket as follows. Multiply $\langle D\rangle$ by with $(-A)^{-3 w(D)}$, where $w(D)$ is the writhe of $D$, and then substitute $A=t^{-1 / 4}$.

Remark 1.4. Theorem 1.3 leads to formulae for the coefficients of the Jones polynomial of a link in terms of topological quantities of the graph $\mathbb{G}_{A}$ corresponding to any diagram of the link [8, 9]. These formulae become particularly effective if $\mathbb{G}_{A}$ corresponds to an $A$ adequate diagram; in particular, Theorem 1.5 below can be recovered from these formulae.

The polynomial $J_{K}(t)$ fits within a family of knot polynomials known as the colored Jones polynomials. A convenient way to express this family is in terms of Chebyshev polynomials. For $n \geq 0$, the polynomial $S_{n}(x)$ is defined recursively as follows:

$$
S_{n+1}=x S_{n}-S_{n-1}, \quad S_{1}(x)=x, \quad S_{0}(x)=1 .
$$

Let $D$ be a diagram of a link $K$. For an integer $m>0$, let $D^{m}$ denote the diagram obtained from $D$ by taking $m$ parallel copies of $K$. This is the $m$-cable of $D$ using the blackboard framing; if $m=1$ then $D^{1}=D$. Let $\left\langle D^{m}\right\rangle$ denote the Kauffman bracket of $D^{m}$ and let $w=w(D)$ denote the writhe of $D$. Then we may define the function

$$
G(n+1, A):=\left((-1)^{n} A^{n^{2}+2 n}\right)^{-w}(-1)^{n-1}\left(\frac{A^{4}-A^{-4}}{A^{2 n}-A^{-2 n}}\right)\left\langle S_{n}(D)\right\rangle,
$$

where $S_{n}(D)$ is a linear combination of blackboard cablings of $D$, obtained via equation (11), and the notation $\left\langle S_{n}(D)\right\rangle$ means extend the Kaufmann bracket linearly. That is, for diagrams $D_{1}$ and $D_{2}$ and scalars $a_{1}$ and $b_{1},\left\langle a_{1} D_{1}+a_{2} D_{2}\right\rangle=a_{1}\left\langle D_{1}\right\rangle+a_{2}\left\langle D_{2}\right\rangle$. Finally, the reduced $n$-th colored Jones polynomial of $K$, denoted

$$
J_{K}^{n}(t)=\alpha_{n} t^{j(n)}+\beta_{n} t^{j(n)-1}+\ldots+\beta_{n}^{\prime} t^{j^{\prime}(n)+1}+\alpha_{n}^{\prime} t^{j^{\prime}(n)},
$$

is obtained from $G(n, A)$ by substituting $t:=A^{-4}$.

For a given a diagram $D$ of $K$, there is a lower bound for $j^{\prime}(n)$ in terms of data about the state graph $\mathbb{G}_{A}$, and this bound is sharp when $D$ is $A$-adequate. Similarly, there is an upper bound on $j(n)$ in terms of $\mathbb{G}_{B}$ that is realized when $D$ is $B$-adequate [30. See Theorem 2.5 for a related statement. In addition, Dasbach and Lin showed that for $A$ and $B$-adequate diagrams, the extreme coefficients of $J_{K}^{n}(t)$ have a particularly nice form. 

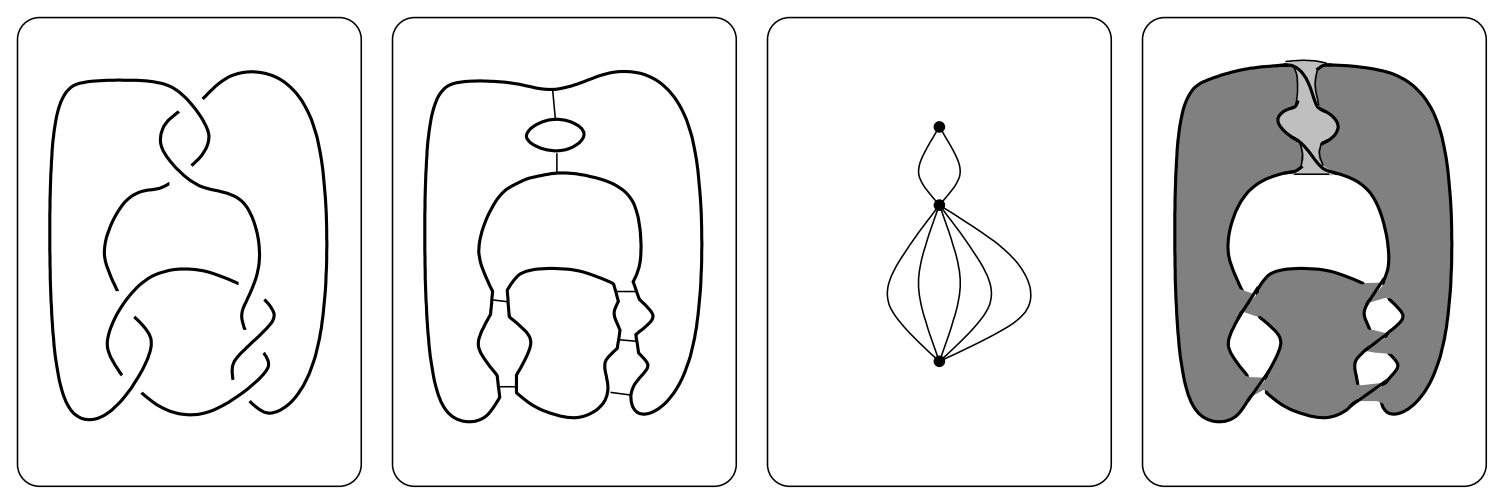

Figure 4. Left to right: A diagram. The graphs $H_{A}$ and $\mathbb{G}_{A}$. The state surface $S_{A}$.

Theorem 1.5 ([10]). If $D$ is an $A$-adequate diagram, then $\alpha_{n}^{\prime}$ and $\beta_{n}^{\prime}$ are independent of $n>1$. In particular, $\left|\alpha_{n}^{\prime}\right|=1$ and $\left|\beta_{n}^{\prime}\right|=1-\chi\left(\mathbb{G}_{A}^{\prime}\right)$, where $\mathbb{G}_{A}^{\prime}$ is the reduced graph.

Similarly, if $D$ is $B$-adequate, then $\left|\alpha_{n}\right|=1$ and $\left|\beta_{n}\right|=1-\chi\left(\mathbb{G}_{B}^{\prime}\right)$.

Now the following definition makes sense in the light of Theorem 1.5.

Definition 1.6. For an $A$-adequate link $K$, we define the stable penultimate coefficient of $J_{K}^{n}(t)$ to be $\beta_{K}^{\prime}:=\left|\beta_{n}^{\prime}\right|$, for $n>1$.

Similarly, for a $B$-adequate link $K$, we define the stable second coefficient of $J_{K}^{n}(t)$ to be $\beta_{K}:=\left|\beta_{n}\right|$, for $n>1$.

For example, in Figure 2, $\mathbb{G}_{A}^{\prime}$ is a tree. Thus, for the link in the figure, $\beta_{K}^{\prime}=0$.

Remark 1.7. It is known that in general, the colored Jones polynomials $J_{K}^{n}(t)$ satisfy linear recursive relations in $n$ [21. In this setting, the properties stated in Theorem 1.5 can be thought of as strong manifestations of the general recursive phenomena, under the hypothesis of adequacy. For arbitrary knots the coefficients $\left|\beta_{n}\right|,\left|\beta_{n}^{\prime}\right|$ do not, in general, stabilize. For example, for $q>p>2$, the coefficients $\left|\beta_{n}\right|,\left|\beta_{n}^{\prime}\right|$ of the $(p, q)$ torus link are periodic with period 2 (see [3]):

$$
\left|\beta_{n}\right|=\left|\beta_{n+2 k}\right| \quad \text { and } \quad\left|\beta_{n}^{\prime}\right|=\left|\beta_{n+2 k}^{\prime}\right|, \quad \text { for } n \geq 2, k \in \mathbb{N} \text {. }
$$

See of [19, Chapter 10] for more discussion and questions on these periodicity phenomena.

\section{State SuRfaces}

In this section, we consider 2-dimensional objects: namely, certain surfaces associated to Kauffman states. This surface is constructed as follows. Recall that a Kauffman state $\sigma$ gives rise to a collection of circles embedded in the projection plane $S^{2}$. Each of these circles bounds a disk in the ball below the projection plane, where the collection of disks is unique up to isotopy in the ball. Now, at each crossing of $D$, we connect the pair of neighboring disks by a half-twisted band to construct a state surface $S_{\sigma} \subset S^{3}$ whose boundary is $K$. See Figure 4 for an example where $\sigma$ is the all- $A$ state. 
Well-known examples of state surfaces include Seifert surfaces (where the corresponding state $\sigma$ is defined by following an orientation on $K$ ) and checkerboard surfaces for alternating links (where the corresponding state $\sigma$ is either the all- $A$ or all- $B$ state). In this paper, we focus on the all $-A$ and all $-B$ states of a diagram, but we do not require our diagrams to be alternating.

Our surfaces also generalize checkerboard surfaces in the following sense. For an alternating diagram $D$, the white and shaded checkerboard surfaces are $S_{A}$ and $S_{B}$ of the all- $A$ and all $-B$ states. These surfaces can be simultaneously embedded in $S^{3}$ so that their intersection consists of disjoint segments, one at each crossing. Collapsing each of these segments to a point will map $S_{A} \cup S_{B}$ to the projection sphere, which is the Turaev surface $F(D)$ associated to an alternating diagram.

In our more general setting, suppose that we modify the surfaces $S_{A}$ and $S_{B}$ so that $S_{A}$ is constructed out of disks in the 3-ball above the projection plane, while $S_{B}$ is constructed out of disks in the 3-ball below the projection plane. (See Figure 3 for the boundaries of these disks.) Then, once again, $S_{A} \cap S_{B}$ will consist of disjoint segments at the crossings, and collapsing each segment to a point will map $S_{A} \cup S_{B}$ to the Turaev surface $F(D)$. Informally, each of $S_{A}$ and $S_{B}$ forms "half" of the Turaev surface, just as each checkerboard surface of an alternating diagram forms "half" of the projection plane.

In general, the graph $\mathbb{G}_{\sigma}$ has the following relationship to the state surface $S_{\sigma}$.

Lemma 2.1. The graph $\mathbb{G}_{\sigma}$ is a spine for the surface $S_{\sigma}$.

Proof. By construction, $\mathbb{G}_{\sigma}$ has one vertex for every circe of $s_{\sigma}$ (hence every disk in $S_{\sigma}$ ), and one edge for every half-twisted band in $S_{\sigma}$. This gives a natural embedding of $\mathbb{G}_{\sigma}$ into the surface, where every vertex is embedded into the corresponding disk, and every edge runs through the corresponding half-twisted band. This gives a spine for $S_{\sigma}$.

The surfaces $S_{\sigma}$ are, in general, non-orientable (checkerboard surfaces already exhibit this phenomenon). The state graph $\mathbb{G}_{\sigma}$ encodes orientability via the following criterion, whose proof we leave as a pleasant exercise.

Lemma 2.2. The surface $S_{\sigma}$ is orientable if and only if $\mathbb{G}_{\sigma}$ is bipartite.

We need the following definition.

Definition 2.3. Let $M$ be an orientable 3-manifold and $S \subset M$ a properly embedded surface. We say that $S$ is essential in $M$ if the boundary of a regular neighborhood of $S$, denoted $\widetilde{S}$, is incompressible and boundary-incompressible. If $S$ is orientable, then $\widetilde{S}$ consists of two copies of $S$, and the definition is equivalent to the standard notion of "incompressible and boundary-incompressible." If $S$ is non-orientable, this is equivalent to $\pi_{1}$-injectivity of $S$, the stronger of two possible senses of incompressibility.

The state surfaces surface $S_{\sigma}$ are often non-orientable. In this case, $S^{3} \backslash \backslash \widetilde{S_{\sigma}}$ is the disjoint union of $M_{A}=S^{3} \backslash \backslash S_{\sigma}$ and a twisted $I$-bundle over $S_{\sigma}$.

Again, we are especially interested in the state surfaces of the all- $A$ and all $-B$ states. For these states, there is a particularly nice relationship between the state surface $S_{\sigma}$ and the state graph $\mathbb{G}_{\sigma}$. 
Theorem 2.4 (Ozawa 37]). Let $D(K)$ be a diagram of a link $K$, and let $\sigma$ be the all- $A$ or all-B state. Then the state surface $S_{\sigma}$ is essential in $S^{3} \backslash K$ if and only if $\mathbb{G}_{\sigma}$ contains no 1-edge loops.

In fact, Ozawa's theorem also applies to a number of other states, which he calls $\sigma-$ homogeneous 37.

Ozawa proves Theorem 2.4 by decomposing the diagram into tangles so that $S_{\sigma}$ is a Murasugi sum. We have an alternate proof of this result in [19] that uses a decomposition of the complement of $S_{\sigma}$ into topological balls. We will discuss this more in Section 5 .

2.1. Colored Jones polynomials and slopes of state surfaces. Garoufalidis has conjectured that for a knot $K$, the growth of the degree of the colored Jones polynomial is related to essential surfaces in the manifold $S^{3} \backslash K$ [20]. In [18], we show that this holds for $A$-adequate diagrams of a knot $K$ and the essential surface $S_{A}$. In this subsection, we review these results.

Given $K \subset S^{3}$, let $M=M_{K}$ denote the compact 3-manifold created when a tubular neighborhood of $K$ is removed from $S^{3}$. There is a canonical meridian-longitude basis of $H_{1}(\partial M)$, which we denote by $\langle\mu, \lambda\rangle$. Any properly embedded surface $(S, \partial S) \subset(M, \partial M)$ has $S \cap \partial M$ a simple closed curve on $\partial M$. The homology class of $\partial S$ in $H_{1}(\partial M)$ is determined by an element $p / q \in \mathbb{Q} \cup\{1 / 0\}$ : the slope of $S$. An element $p / q \in \mathbb{Q} \cup\{1 / 0\}$ is called a boundary slope of $K$ if there is a properly embedded essential surface $(S, \partial S) \subset$ $(M, \partial M)$, such that $\partial S$ is homologous to $p \mu+q \lambda \in H_{1}(\partial M)$. Hatcher has shown that every knot $K \subset S^{3}$ has finitely many boundary slopes 23 .

Let $j(n)$ denote the highest degree of $J_{K}(n, t)$ in $t$, and let $j^{\prime}(n)$ denote the lowest degree. Consider the sequences

$$
j s_{K}:=\left\{\frac{4 j(n)}{n^{2}}: n>0\right\} \quad \text { and } \quad j s_{K}^{\prime}:=\left\{\frac{4 j^{\prime}(n)}{n^{2}}: n>0\right\} .
$$

Garoufalidis has conjectured [20] that for each knot $K$, every cluster point (i.e., every limit of a subsequence) of $j s_{K}$ or $j s_{K}^{*}$ is a boundary slope of $K$. In [18, the authors proved this is true for $A$-adequate knots, and the boundary slope comes from the incompressible surface $S_{A}$. This is the content of the following theorem.
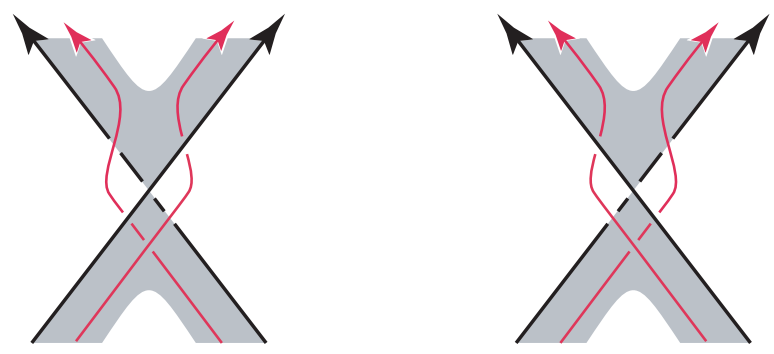

FIgURE 5. Left: a positive crossing, and a piece of $S_{B}$ near the crossing. Locally, this crossing contributes +2 to the slope of $S_{B}$, and makes no contribution to the slope of $S_{A}$. Right: a negative crossing contributes -2 to the slope of $S_{A}$, and makes no contribution to the slope of $S_{B}$. 
Theorem 2.5 ([18]). Let $D$ be an $A$-adequate diagram of a knot $K$ and let $b\left(S_{A}\right) \in \mathbb{Z}$ denote the boundary slope of the essential surface $S_{A}$. Then

$$
\lim _{n \rightarrow \infty} \frac{4 j^{\prime}(n)}{n^{2}}=b\left(S_{A}\right)=-2 c_{-},
$$

where $c_{-}$is the number of negative crossings in D. (See Figure 5 , right.)

Similarly, if $D$ is a $B$-adequate diagram of a knot $K$, let $b\left(S_{B}\right) \in \mathbb{Z}$ denote the boundary slope of the essential surface $S_{B}$. Then

$$
\lim _{n \rightarrow \infty} \frac{4 j(n)}{n^{2}}=b\left(S_{B}\right)=-2 c_{+},
$$

where $c_{+}$is the number of positive crossings in D. (See Figure 5, left.)

Additional families of knots for which the conjecture is true are given by Garoufalidis [20] and more recently by Dunfeld and Garoufalidis [13].

\section{Cutting along the state surface}

In this section, we focus on the 3-manifold formed by cutting along the state surface $S_{A}$. Using its 3-dimensional structure, we will relate the hyperbolic geometry of $S^{3} \backslash K$ to the Jones and colored Jones polynomials of $K$.

\subsection{Geometry and topology of the state surface complement.}

Definition 3.1. Let $K \subset S^{3}$ be a link, and $S_{A}$ the all- $A$ state surface. We let $M$ denote the link complement, $M=S^{3} \backslash K$, and we let $M_{A}:=M \backslash \backslash S_{A}$ denote the path-metric closure of $M \backslash S_{A}$. Note that $M_{A}=\left(S^{3} \backslash K\right) \backslash \backslash S_{A}$ is homeomorphic to $S^{3} \backslash \backslash S_{A}$, obtained by removing a regular neighborhood of $S_{A}$ from $S^{3}$.

We will refer to $P=\partial M_{A} \cap \partial M$ as the parabolic locus of $M_{A}$; it consists of annuli. The remaining, non-parabolic boundary $\partial M_{A} \backslash \partial M$ is the unit normal bundle of $S_{A}$.

Our goal is to use the state graph $\mathbb{G}_{A}$ to understand the topological structure of $M_{A}$. One result along these lines is a straightforward characterization of when $S_{A}$ is a fiber surface for $S^{3} \backslash K$, or equivalently when $M_{A}$ is an $I$-bundle over $S_{A}$.

Theorem 3.2. Let $D(K)$ be any link diagram, and let $S_{A}$ be the spanning surface determined by the all-A state of this diagram. Then the following are equivalent:

(1) The reduced graph $\mathbb{G}_{A}^{\prime}$ is a tree.

(2) $S^{3} \backslash K$ fibers over $S^{1}$, with fiber $S_{A}$.

(3) $M_{A}=S^{3} \backslash \backslash S_{A}$ is an I-bundle over $S_{A}$.

For example, in the link diagram depicted in Figure 4, the graph $\mathbb{G}_{A}^{\prime}$ is a tree with two edges. Thus the state surface $S_{A}$ shown in Figure 4 is a fiber in $S^{3} \backslash K$.

In general, one may apply the annulus version of the JSJ decomposition theory [25, 26] to cut $M_{A}$ into three types of pieces: $I$-bundles over sub-surfaces of $S_{A}$, Seifert fibered spaces that are solid tori, and guts, i.e. the portion that admits a hyperbolic metric.

The pieces of the JSJ decomposition give significant information about the manifold $M_{A}$. For example, if $\operatorname{guts}\left(M_{A}\right)=\emptyset$, then $M_{A}$ is a union of $I$-bundles and solid tori. Such an $M_{A}$ is called a book of $I$-bundles, and the surface $S_{A}$ is called a fibroid [7]. 
The guts of $M_{A}$ are a good measurement of topological complexity. To express this more precisely, we need the following definition.

Definition 3.3. Let $Y$ be a compact cell complex with connected components $Y_{1}, \ldots, Y_{n}$. Let $\chi(\cdot)$ denote the Euler characteristic. This can be split into positive and negative parts, with notation borrowed from the Thurston norm [43]:

$$
\chi_{+}(Y)=\sum_{i=1}^{n} \max \left\{\chi\left(Y_{i}\right), 0\right\}, \quad \chi_{-}(Y)=\sum_{i=1}^{n} \max \left\{-\chi\left(Y_{i}\right), 0\right\} .
$$

Note that $\chi(Y)=\chi_{+}(Y)-\chi_{-}(Y)$. In the case that $Y=\emptyset$, we have $\chi_{+}(\emptyset)=\chi_{-}(\emptyset)=0$.

The negative Euler characteristic $\chi_{-}\left(\operatorname{guts}\left(M_{A}\right)\right)$ serves as a useful measurement of how far $S_{A}$ is from being a fiber or a fibroid in $S^{3} \backslash K$. In addition, it relates to the volume of $M_{A}$. The following theorem was proved by Agol, Storm, and Thurston.

Theorem 3.4 (Theorem 9.1 of [2]). Let $M$ be finite-volume hyperbolic 3-manifold, and let $S \subset M$ be a properly embedded essential surface. Then

$$
\operatorname{vol}(M) \geq v_{8} \chi_{-}(\operatorname{guts}(M \backslash \backslash S)),
$$

where $v_{8}=3.6638 \ldots$ is the volume of a regular ideal octahedron.

We apply Theorem 3.4 to the essential surface $S_{A}$ for a prime, $A$-adequate diagram of a hyperbolic link. In order to do so, we develop techniques for determining the Euler characteristic of the guts of $S_{A}$. We find that it can be read off of a diagram of the link.

Theorem 3.5. Let $D(K)$ be an $A$-adequate diagram, and let $S_{A}$ be the essential spanning surface determined by this diagram. Then

$$
\chi_{-}\left(\operatorname{guts}\left(S^{3} \backslash \backslash S_{A}\right)\right)=\chi_{-}\left(\mathbb{G}_{A}^{\prime}\right)-\left\|E_{c}\right\|,
$$

where $\left\|E_{c}\right\| \geq 0$ is a diagrammatic quantity.

The quantity $\left\|E_{c}\right\|$ is the number of complex essential product disks (EPDs). We will give its definition and examples in the next subsection. For now, we point out that in many cases, the quantity $\left\|E_{c}\right\|$ vanishes. For example, this happens for alternating links [29], as well as for most Montesinos links [19, Corollary 9.21].

When we combine Theorems 3.4 and 3.5, and recall that $S^{3} \backslash \backslash S_{A}$ is homeomorphic to $\left(S^{3} \backslash K\right) \backslash \backslash S_{A}$, we obtain

$$
\operatorname{vol}\left(S^{3} \backslash K\right) \geq v_{8} \chi_{-}\left(\operatorname{guts}\left(S^{3} \backslash \backslash S_{A}\right)\right)=\chi_{-}\left(\mathbb{G}_{A}^{\prime}\right)-\left\|E_{c}\right\|,
$$

where the equality comes from Theorem 3.5. This leads to the following.

Theorem 3.6. Let $D=D(K)$ be a prime A-adequate diagram of a hyperbolic link $K$. Then

$$
\operatorname{vol}\left(S^{3} \backslash K\right) \geq v_{8}\left(\chi_{-}\left(\mathbb{G}_{A}^{\prime}\right)-\left\|E_{c}\right\|\right),
$$

where $\left\|E_{c}\right\|$ is the same diagrammatic quantity as in the statement of Theorem 3.5, and $v_{8}=3.6638 \ldots$ is the volume of a regular ideal octahedron.

There is a symmetric result for $B$-adequate diagrams. 

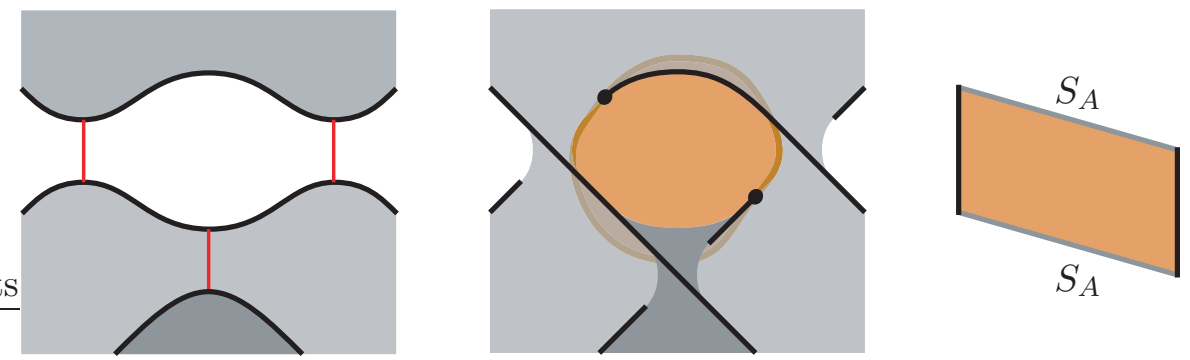

Figure 6. An EPD in $M_{A}$ containing a single 2-edge loop in $\mathbb{G}_{A}$, with edges in different twist regions in the link diagram.

3.2. Essential product disks. We now describe the quantity $\left\|E_{c}\right\|$ more carefully, and discuss how it relates to the graph $\mathbb{G}_{A}$. To begin, we review some terminology.

Definition 3.7. Let $M$ be a 3-manifold with boundary, and with prescribed parabolic locus consisting of annuli. An essential product disk in $M$, or EPD for short, is a properly embedded disk whose boundary has geometric intersection number 2 with the parabolic locus. Note that an EPD is an $I$-bundle over an interval. See Figure 6 for an example of such a disk in $M_{A}=S^{3} \backslash \backslash S_{A}$.

If $B$ is an $I$-bundle in $M$, we say that a collection $\left\{D_{1}, \ldots, D_{n}\right\}$ of disjoint EPDs spans $B$ if their complement in $B$ is a disjoin union of solid tori and 3-balls.

Essential product disks are integral to understanding the size of guts $\left(M_{A}\right)$. In particular, the proof of Theorem 3.5 requires calculating the Euler characteristic of all the $I$-bundle components in the JSJ decomposition of $M_{A}=S^{3} \backslash \backslash S_{A}$. To do this, we show that each component of the $I$-bundle is spanned by EPDs, and find a particular spanning set. (See Theorem 5.6 in Section 5.)

Definition 3.8. Two crossings in $D$ are defined to be twist equivalent if there is a simple closed curve in the projection plane that meets $D$ at exactly those two crossings. The diagram is called twist reduced if every equivalence class of crossings is a twist region (a chain of crossings between two strands of $K$ ). The number of equivalence classes is denoted $t(D)$, the twist number of $D$.

Every twist region in $D(K)$ with at least two crossings gives rise to EPDs. For instance, in Figure 7 , there are three crossings in the twist region. The boundary of each EPD shown lies on the state surface $S_{A}$, and crosses the knot diagram exactly twice. Note there are two EPDs that encircle one bigon each, and one EPD that encircles two bigons. Any two of these will suffice in a spanning set.

The essential product disk in Figure 6 does not lie in a single twist region. For another example that does not lie in a single twist region, see Figure 8. Note that in each of Figures 6, 7] and 8, the EPD can be naturally associated to one or more 2-edge loops in the state graph $\mathbb{G}_{A}$.

In Figure 8, the EPD exhibits more complicated behavior than in the other examples, in that it bounds nontrivial portions of the graph $H_{A}$. We call an EPD that bounds nontrivial portions of $H_{A}$ on both sides a complex EPD. The minimal number of complex 

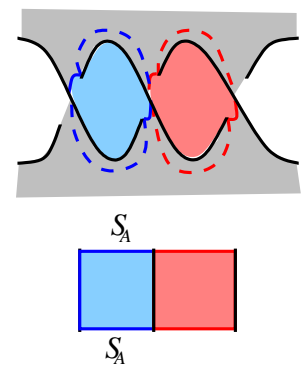

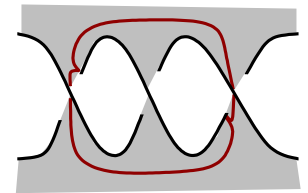

$S_{A}$

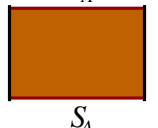

Figure 7. Shown are three EPDs in a twist region.
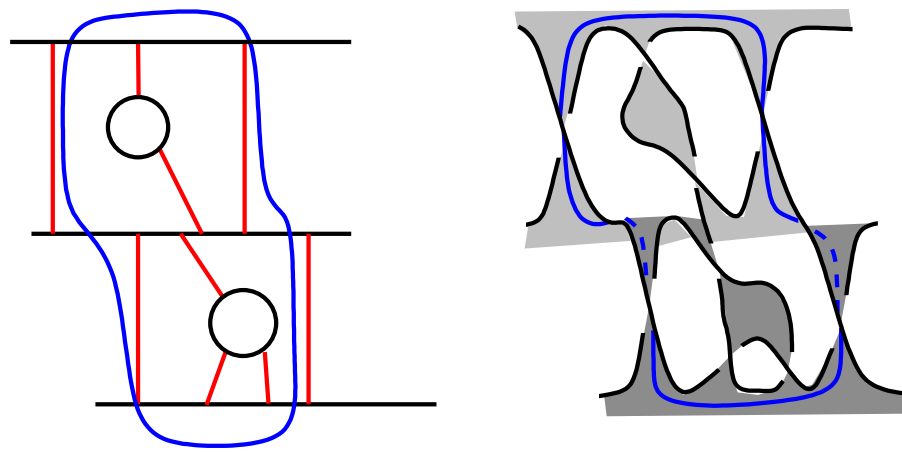

Figure 8. An EPD in $M_{A}$ containing two 2-edge loops of $\mathbb{G}_{A}$.

EPDs in the spanning set of the maximal $I$-bundle of $M_{A}$ is denoted $\left\|E_{c}\right\|$, and is exactly the correction term in Theorem 3.5. By analyzing EPDs, we show the following.

Proposition 3.9. If $D$ is prime and $A$-adequate, such that every 2 -edge loop in $\mathbb{G}_{A}$ has edges belonging to the same twist region, then $\left\|E_{c}\right\|=0$. Hence

$$
\operatorname{vol}\left(S^{3} \backslash K\right) \geq v_{8}\left(\chi_{-}\left(\mathbb{G}_{A}^{\prime}\right)\right)
$$

3.3. Volume estimates. One family of knots and links that satisfies Proposition 3.9 is that of alternating links [29]. If $D=D(K)$ is a prime, twist-reduced alternating link diagram, then it is both $A$ - and $B$-adequate, and for each 2-edge loop in $\mathbb{G}_{A}$ or $\mathbb{G}_{B}$, both edges belong to the same twist region. Theorem 3.6 gives lower bounds on volume in terms of both $\chi_{-}\left(\mathbb{G}_{A}^{\prime}\right)$ and $\chi_{-}\left(\mathbb{G}_{B}^{\prime}\right)$. By averaging these two lower bounds, one recovers Lackenby's lower bound on the volume of hyperbolic alternating links, in terms of the twist number $t(D)$.

Theorem 3.10 (Theorem 2.2 of [2]). Let $D$ be a reduced alternating diagram of a hyperbolic link $K$. Then

$$
\frac{v_{8}}{2}(t(D)-2) \leq \operatorname{vol}\left(S^{3} \backslash K\right)<10 v_{3}(t(D)-1),
$$

where $v_{3}=1.0149 \ldots$ is the volume of a regular ideal tetrahedron and $v_{8}=3.6638 \ldots$ is the volume of a regular ideal octahedron. 
Theorem 3.6 greatly expands the list of manifolds for which we can compute explicitly the Euler characteristic of the guts, and can be used to derive results analogous to Theorem 3.10. As a sample, we state the following.

Theorem 3.11. Let $D(K)$ be a diagram of a hyperbolic link $K$, obtained as the closure of a positive braid with at least three crossings in each twist region. Then

$$
\frac{2 v_{8}}{3} t(D) \leq \operatorname{vol}\left(S^{3} \backslash K\right)<10 v_{3}(t(D)-1),
$$

where $v_{3}=1.0149 \ldots$ is the volume of a regular ideal tetrahedron and $v_{8}=3.6638 \ldots$ is the volume of a regular ideal octahedron.

Observe that the multiplicative constants in the upper and lower bounds differ by a rather small factor of about 4.155 .

We obtain similarly tight two-sided volume bounds for Montesinos links, using these guts techniques [19].

Theorem 3.12. Let $K \subset S^{3}$ be a Montesinos link with a reduced Montesinos diagram $D(K)$. Suppose that $D(K)$ contains at least three positive tangles and at least three negative tangles. Then $K$ is a hyperbolic link, satisfying

$$
\frac{v_{8}}{4}(t(D)-\# K) \leq \operatorname{vol}\left(S^{3} \backslash K\right)<2 v_{8} t(D),
$$

where $v_{8}=3.6638 \ldots$ is the volume of a regular ideal octahedron and $\# K$ is the number of link components of $K$. The upper bound on volume is sharp.

Similar results using different techniques have been obtained by the authors in [14, 15, 16], and by Purcell in [39].

3.4. Relations with the colored Jones polynomial. The main results of [19] explore the idea that the stable coefficient $\beta_{K}^{\prime}$ does an excellent job of measuring the geometric and topological complexity of the manifold $M_{A}=S^{3} \backslash \backslash S_{A}$. (Similarly, $\beta_{K}$ measures the complexity of $M_{B}=S^{3} \backslash \backslash M_{B}$.)

For instance, note that we have $\left|\beta_{K}^{\prime}\right|=1-\chi\left(\mathbb{G}_{A}^{\prime}\right)=0$ exactly when $\chi\left(\mathbb{G}_{A}^{\prime}\right)=1$, or equivalently $\mathbb{G}_{A}^{\prime}$ is a tree. Thus it follows from Theorem 3.2 that $\beta_{K}^{\prime}$ is exactly the obstruction to $S_{A}$ being a fiber.

Corollary 3.13. For an $A$-adequate link $K$, the following are equivalent:

(1) $\beta_{K}^{\prime}=0$.

(2) For every A-adequate diagram of $D(K), S^{3} \backslash K$ fibers over $S^{1}$ with fiber the corresponding state surface $S_{A}=S_{A}(D)$.

(3) For some $A$-adequate diagram $D(K), M_{A}=S^{3} \backslash \backslash S_{A}$ is an $I$-bundle over $S_{A}(D)$.

Similarly, $\left|\beta_{K}^{\prime}\right|=1$ precisely when $S_{A}$ is a fibroid of a particular type [19, Theorem 9.18]. In general, the JSJ decomposition of $M_{A}$ contains guts: the non-trivial hyperbolic pieces. In this case, $\left|\beta_{K}^{\prime}\right|$ measures the complexity of the guts together with certain complicated parts of the maximal $I$-bundle of $M_{A}$. 
Theorem 3.14. Suppose $K$ is an A-adequate link whose stable colored Jones coefficient is $\beta_{K}^{\prime} \neq 0$. Then, for every $A$-adequate diagram $D(K)$,

$$
\chi_{-}\left(\operatorname{guts}\left(M_{A}\right)\right)+|| E_{c}||=\left|\beta_{K}^{\prime}\right|-1 .
$$

Furthermore, if $D$ is prime and every 2-edge loop in $\mathbb{G}_{A}$ has edges belonging to the same twist region, then $\left\|E_{c}\right\|=0$ and

$$
\chi_{-}\left(\operatorname{guts}\left(M_{A}\right)\right)=\left|\beta_{K}^{\prime}\right|-1 .
$$

The volume conjecture of Kashaev and Murakami-Murakami [28, 36] states that all hyperbolic knots satisfy

$$
2 \pi \lim _{n \rightarrow \infty} \frac{\log \left|J_{K}^{n}\left(e^{2 \pi i / n}\right)\right|}{n}=\operatorname{vol}\left(S^{3} \backslash K\right)
$$

If the volume conjecture is true, then for large $n$, there would be a relation between the coefficients of $J_{K}^{n}(t)$ and the volume of the knot complement. In recent years, articles by Dasbach and Lin and the authors have established relations for several classes of knots [11, 14, 15, 16]. However, in all these results, the lower bound involved first showing that the Jones coefficients give a lower bound on twist number, then showing twist number gives a lower bound on volume. Each of these steps is known to fail outside special families of knots [16, 17]. Moreover, the two-step argument is indirect, and the constants produced are not sharp. By contrast, in [19], we bound volume below in terms of $\chi_{-}$(guts), which is directly related to colored Jones coefficients. This yields sharper lower bounds on volumes, along with a more intrinsic explanation for why these lower bounds exist. For instance, Theorems 3.11 and 3.12 have the following corollaries.

Corollary 3.15. Suppose that a hyperbolic link $K$ is the closure of a positive braid with at least three crossings in each twist region. Then

$$
v_{8}\left(\left|\beta_{K}^{\prime}\right|-1\right) \leq \operatorname{vol}\left(S^{3} \backslash K\right)<15 v_{3}\left(\left|\beta_{K}^{\prime}\right|-1\right)-10 v_{3},
$$

where $v_{3}=1.0149 \ldots$ is the volume of a regular ideal tetrahedron and $v_{8}=3.6638 \ldots$ is the volume of a regular ideal octahedron.

Corollary 3.16. Let $K \subset S^{3}$ be a Montesinos link with a reduced Montesinos diagram $D(K)$. Suppose that $D(K)$ contains at least three positive tangles and at least three negative tangles. Then $K$ is a hyperbolic link, satisfying

$$
v_{8}\left(\max \left\{\left|\beta_{K}\right|,\left|\beta_{K}^{\prime}\right|\right\}-1\right) \leq \operatorname{vol}\left(S^{3} \backslash K\right)<4 v_{8}\left(\left|\beta_{K}\right|+\left|\beta_{K}^{\prime}\right|-2\right)+2 v_{8}(\# K),
$$

where $\# K$ is the number of link components of $K$.

\section{A WORKED EXAMPLE}

In this section, we illustrate several of the above theorems on the two-component link of Figure 9. The figure also shows the graphs $H_{A}, \mathbb{G}_{A}$, and $\mathbb{G}_{A}^{\prime}$ for this link diagram.

In this example, it turns out that the manifold $M_{A}=S^{3} \backslash S_{A}$ contains a single essential product disk. This disk $D$ is shown in Figure 10, Observe that this lone EPD corresponds to the single 2 -edge loop in $\mathbb{G}_{A}$. Note as well that collapsing two edges of $\mathbb{G}_{A}$ to a single 


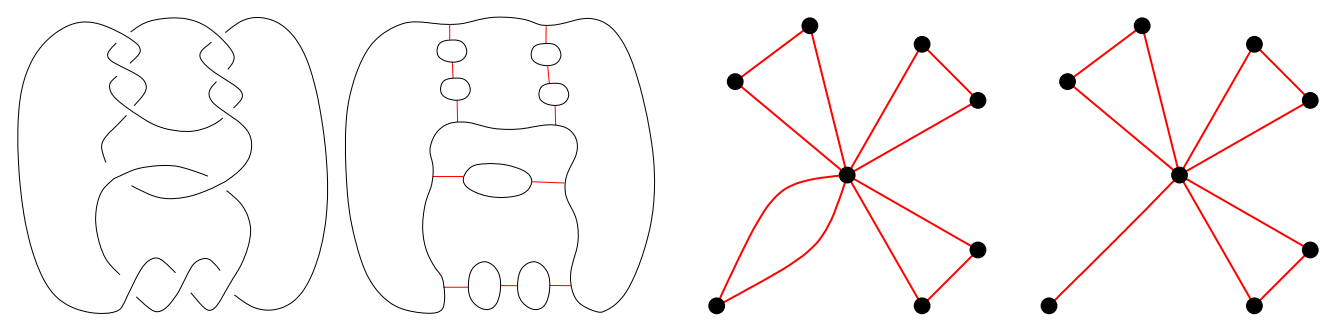

Figure 9. Diagram $D(K)$ of a two-component link, and graphs $H_{A}, \mathbb{G}_{A}$, and $\mathbb{G}_{A}^{\prime}$. All of the discussion in Section 4 pertains to this link.
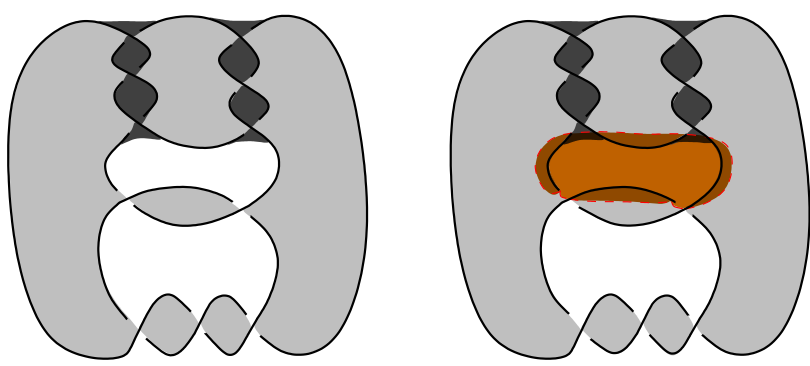

Figure 10. Left: the state surface $S_{A}$ of the link of Figure 9, Right: the single EPD in $M_{A}$ lies below the surface $S_{A}$, and its boundary intersects $K$ at two points in the center of the figure.

edge of $\mathbb{G}_{A}^{\prime}$ changes the Euler characteristic by 1 , while cutting $M_{A}$ along disk $D$ also changes the Euler characteristic by 1 . Thus

$$
\chi_{-}\left(\mathbb{G}_{A}\right)=-\chi\left(\mathbb{G}_{A}\right)=3, \quad \chi_{-}\left(\mathbb{G}_{A}^{\prime}\right)=-\chi\left(\mathbb{G}_{A}^{\prime}\right)=2 .
$$

On the 3-manifold side, recall that $\mathbb{G}_{A}$ is a spine of $S_{A}$. Thus Alexander duality gives

$$
\chi_{-}\left(M_{A}\right)=\chi_{-}\left(S^{3} \backslash \backslash S_{A}\right)=\chi_{-}\left(S_{A}\right)=\chi_{-}\left(\mathbb{G}_{A}\right)=3 .
$$

Because the maximal $I$-bundle of $M_{A}$ is spanned by the single disk $D$, we have

$$
\chi_{-}\left(\operatorname{guts}\left(M_{A}\right)\right)=\chi_{-}\left(M_{A}\right)-1=2=\chi_{-}\left(\mathbb{G}_{A}^{\prime}\right),
$$

exactly as predicted by Theorem 3.5 with $\left\|E_{c}\right\|=0$.

By Theorem 3.4. $\chi_{-}\left(\operatorname{guts}\left(M_{A}\right)\right)$ also gives a lower bound on the hyperbolic volume of $S^{3} \backslash K$. In this example,

$$
\chi_{-}\left(\operatorname{guts}\left(M_{A}\right)\right)=\chi_{-}\left(\mathbb{G}_{A}^{\prime}\right)=\left|\beta_{K}^{\prime}\right|-1=2,
$$

so the lower bound is $2 v_{8} \approx 7.3276$. Meanwhile, the actual hyperbolic volume of the link in Figure 9 is $\operatorname{vol}\left(S^{3} \backslash K\right) \approx 11.3407$.

Turning to the all- $B$ resolution, Figure 11 shows the graphs $H_{B}, \mathbb{G}_{B}$, and $\mathbb{G}_{B}^{\prime}$, as well as the state surface $S_{B}$. This time, $\mathbb{G}_{B}^{\prime}$ is a tree, thus Theorem 3.2 (applied to a reflected diagram) implies $S_{B}$ is a fiber.

One important point to note is that even though $S^{3} \backslash K$ is fibered, it does contain surfaces (such as $S_{A}$ ) with quite a lot of guts. Conversely, having $\left|\beta_{K}^{\prime}\right|>0$, as we do here, 

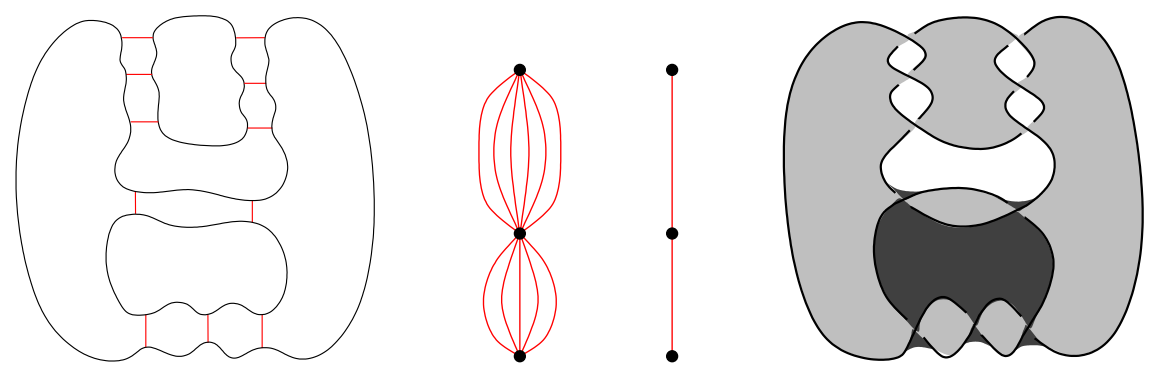

Figure 11. The graphs $H_{B}, \mathbb{G}_{B}$, and $\mathbb{G}_{B}^{\prime}$ for the link of Figure 9, and the corresponding surface $S_{B}$.

only means that the surface $S_{A}$ is not a fiber - it does not rule out $S^{3} \backslash K$ being fibered in another way, as indeed it is.

\section{A ClOSER LOOK AT THE POLYHEDRAL DECOMPOSITION}

To prove all the results that were surveyed in Section 3, we cut $M_{A}$ along a collection of disks, to obtain a decomposition of $M_{A}$ into ideal polyhedra. Here, a (combinatorial) ideal polyhedron is a 3-ball with a graph on its boundary, such that complementary regions of the graph are simply connected, and the vertices have been removed (i.e. lie at infinity).

Our decomposition is a generalization of Menasco's well-known polyhedral decomposition [32. Menasco's work uses a link diagram to decompose any link complement into ideal polyhedra. When the diagram is alternating, the resulting polyhedra have several nice properties: they are checkerboard colored, with 4-valent vertices, and a well-understood gluing. For alternating diagrams, our polyhedra will be exactly the same as Menasco's. More generally, we will see that our polyhedral decomposition of $M_{A}$ also has a checkerboard coloring and $4-$ valent vertices.

5.1. Cutting along disks. To begin, we need to visualize the state surface $S_{A}$ more carefully. We constructed $S_{A}$ by first, taking a collection of disks bounded by state circles, and then attaching bands at crossings. Recall we ensured the disks were below the projection plane. We visualize the disks as soup cans. That is, for each, a long cylinder runs deep under the projection plane with a disk at the bottom. Soup cans will be nested, with outer state circles bounding deeper, wider soup cans. Isotope the diagram so that it lies on the projection plane, except at crossings which run through a crossing ball. When we are finished, the surface $S_{A}$ lies below the projection plane, except for bands that run through a small crossing ball.

In Figure 12, the state surface for this example is shown lying below the projection plane (although soup cans have been smoothed off at their sharp edges in this figure).

We cut $M_{A}=S^{3} \backslash \backslash S_{A}$ along disks. These disks come from complementary regions of the graph of the link diagram on the projection plane. Notice that each such region corresponds to a complementary region of the graph $H_{A}$, the graph of the $A$-resolution. To form the disk that we cut along, we isotope the disk by pushing it under the projection plane slightly, keeping its boundary on the state surface $S_{A}$, so that it meets the link a

minimal number of times. Indeed, because $S_{A}$ itself lies on or below the projection plane 


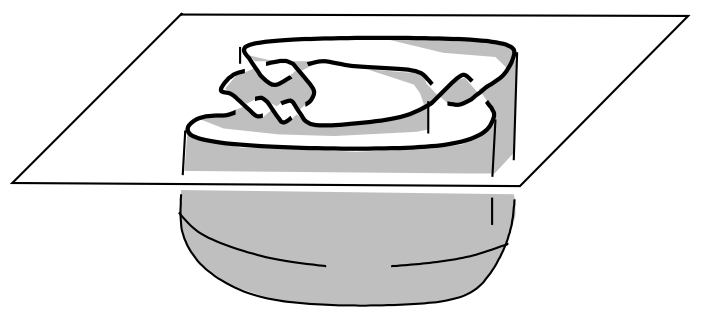

Figure 12. The surface $S_{A}$ is hanging below the projection plane.
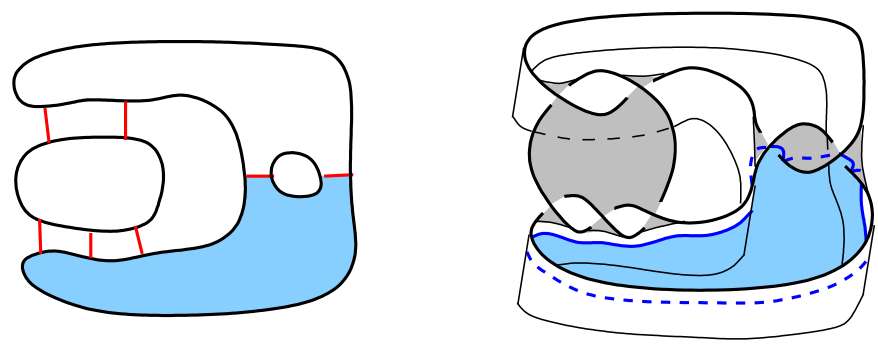

Figure 13. A region of $H_{A}$ together with the corresponding white disk lying just below the projection plane, with boundary (dashed line) on underside of shaded surface.

except in the crossing balls, we can push the disk below the projection plane everywhere except possibly along half-twisted rectangles at the crossings. By further isotopy we can arrange each disk so that its boundary runs meets the link only inside the crossing ball. These isotoped disks are called white disks.

For each region of the complement of $H_{A}$, we have a white disk that meets the link only in crossing balls, and then only at under-crossings. The disk lies slightly below the projection plane everywhere. Figure 13 gives an example.

Some of the white disks will not meet the link at all. These disks are isotopic to soup cans on $S_{A}$; that is, they are innermost disks. We will remove all such white disks from consideration. When we do so, the collection of all remaining white disks, denoted $\mathcal{W}$, consists of those with boundary on the state surface $S_{A}$ and on the link $K$.

It is actually straightforward to see that the components of $M_{A} \backslash \backslash \mathcal{W}$ are 3 -balls, as follows. There will be a single component above the projection plane. Since we cut along each region of the projection graph, either along a disk of $\mathcal{W}$ or an innermost soup can, this component above the projection plane must be homeomorphic to a ball. As for components which lie below the projection plane, these lie between soup can disks. Since any such disk cuts the 3-ball below the projection plane into 3-balls, these components must also each be homeomorphic to 3 -balls. In fact, we know more:

Theorem 5.1 (Theorem 3.12 of [19]). Each component of $M_{A} \backslash \backslash \mathcal{W}$ is an ideal polyhedron with 4-valent ideal vertices and faces colored in a checkerboard fashion: the white faces 
are the disks of $\mathcal{W}$, and the shaded faces contain, but are not restricted to, the innermost disks.

The edges of the ideal polyhedron are given by the intersection of white disks in $\mathcal{W}$ with (the boundary of a regular neighborhood of) $S_{A}$. Each edge runs between strands of the link. The ideal vertices lie on the torus boundary of the tubular neighborhood of a link component. The regions (faces) come from white disks (white faces) and portions of the surface $S_{A}$, which we shade.

Note that each edge bounds a white disk in $\mathcal{W}$ on one side, and a portion of the shaded surface $S_{A}$ on the other side. Thus, by construction, we have a checkerboard coloring of the 2-dimensional regions of our decomposition. Since the white regions are known to be disks, showing that our 3-balls are actually polyhedra amounts to showing that the shaded regions are also simply connected. Showing this requires some work, and the hypothesis of $A$-adequacy is heavily used. The interested reader is referred to [19, Chapter 3] for the details.

5.2. Combinatorial descriptions of the polyhedra. We need a simpler description of our polyhedra than that afforded by the 3-dimensional pictures of the last subsection. We obtain 2-dimensional descriptions in terms of how the white and shaded faces are super-imposed on the projection plane, and how these faces interact with the planar graph $H_{A}$. These descriptions are the starting point for our proofs in [19]. In this subsection we briefly highlight the main characteristics of these descriptions.

Note that in the figures, we often use different colors to indicate different shaded faces. All these colored regions come from the surface $S_{A}$.

5.2.1. Lower polyhedra. The lower polyhedra come from regions bounded between soup cans. Recall that $s_{A}$ denotes the union of state circles of the all- $A$ resolution (i.e. without the added edges of $H_{A}$ corresponding to crossings). As a result, the regions bounded by soup cans will be in one-to-one correspondence with non-trivial components of the complement of $s_{A}$.

Given a lower polyhedron, let $R$ denote the corresponding non-trivial component of the complement of $s_{A}$. The white faces of the polyhedron will correspond to the non-trivial regions of $H_{A}$ in $R$. Since these white faces lie below the projection plane, except in crossing balls, the only portion of the knot that is visible from inside a lower polyhedron is a small segment of a crossing ball. This results in the following combinatorial description.

- Ideal edges of the lower polyhedra run from crossing to crossing.

- Ideal vertices correspond to crossings. At each crossing, two ideal edges bounding a disk from one non-trivial region of $H_{A}$ meet two ideal edges bounding a disk from another non-trivial region (on the opposite side of the crossing). Thus the vertices are 4-valent.

- Shaded faces correspond exactly to soup cans.

As a result, each lower polyhedron is combinatorially identical to the checkerboard polyhedron of an alternating sub-diagram of $D(K)$, where the sub-diagram corresponds to region $R$. This is illustrated in Figure 14, for the knot diagram of Figure 4 ,

Schematically, to sketch a lower polyhedron, start by drawing a portion of $H_{A}$ which lies inside a nontrivial region of the complement of $s_{A}$. Mark an ideal vertex at the center 

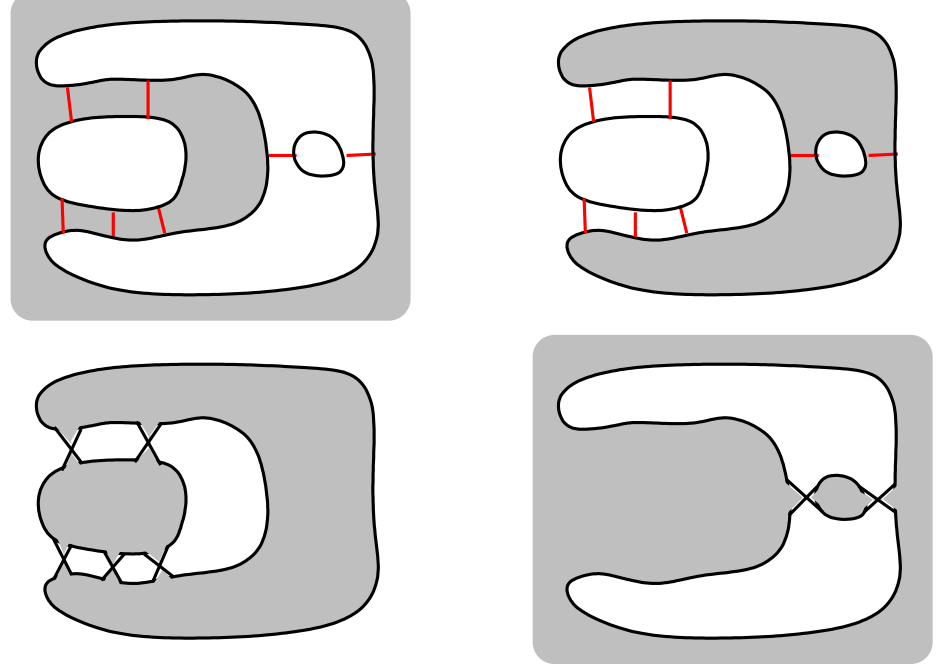

Figure 14. Top row: The two non-trivial regions of the graph $H_{A}$ of Figure 4, Second row: The corresponding lower polyhedra.
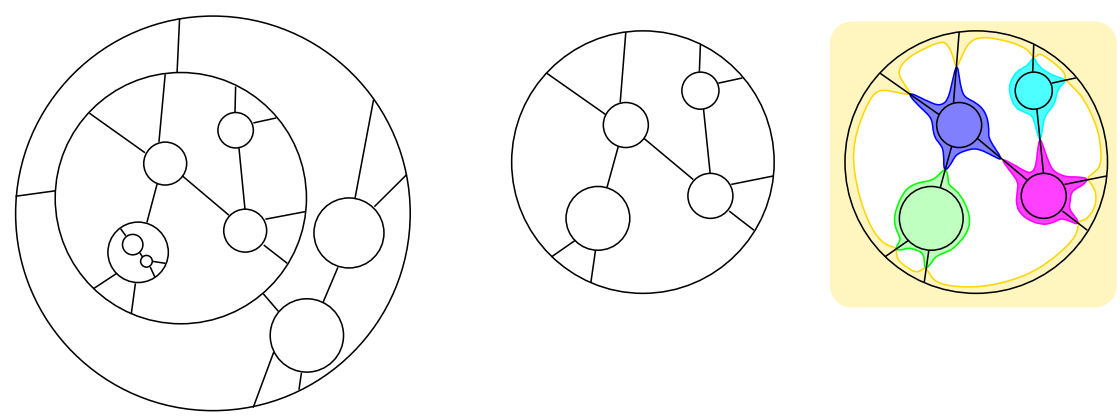

Figure 15. Left to right: An example graph $H_{A}$. A subgraph corresponding to a region of the complement of $s_{A}$. White and shaded faces of the corresponding lower polyhedron.

of each segment of $H_{A}$. Connect these dots by edges bounding white disks, as in Figure 15 .

5.2.2. The upper polyhedron. On the upper polyhedron, ideal vertices correspond to strands of the link visible from inside the upper 3-ball. Since we cut along white faces and the surface $S_{A}$, both of which lie below the projection plane except at crossings, the upper polyhedron can "see" the entire link diagram except for small portions cut off at each undercrossing.

Thus, ideal vertices of the upper polyhedron correspond to strands of the link between undercrossings. In Figure 16, the surface $S_{A}$ is shown in green and gold. There are four ideal edges meeting a crossing, labeled $e_{1}$ through $e_{4}$ in this figure. There are actually three ideal vertices in the figure: one is unlabeled, corresponding to the strand running 


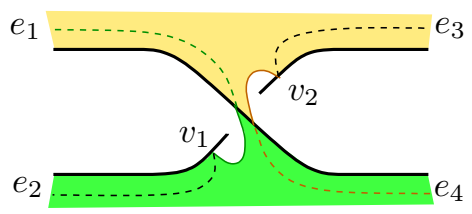

FiguRE 16. Shown are portions of four ideal edges, terminating at undercrossings on a single crossing. Ideal edges $e_{1}$ and $e_{2}$ bound the same white disk and terminate at the ideal vertex $v_{1}$. Ideal edges $e_{3}$ and $e_{4}$ bound the same white disk and terminate at the ideal vertex $v_{2}$. Figure first appeared in [19].
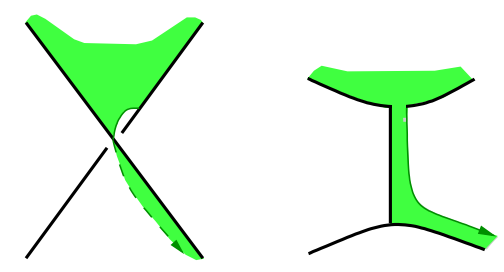

Figure 17. Left: A tentacle continues a shaded face in the upper 3-ball. Right: visualization of the tentacle on the graph $H_{A}$.

over the crossing, and two are labeled $v_{1}$ and $v_{2}$, corresponding to the strands running into the undercrossing.

The surface $S_{A}$ runs through the crossing in a twisted rectangle. Looking again at Figure 16. note that the gold portion of $S_{A}$ at the top of that figure is not cut off at the crossing by an ideal edge terminating at $v_{2}$. Instead, it follows $e_{4}$ through the crossing and along the underside of the figure, between $e_{4}$ and the link. Similarly for the green portion of $S_{A}$ : it follows the edge $e_{1}$ through the crossing and continues between the edge and the link.

We visualize these thin portions of shaded face between an edge and the link as tentacles, and sketch them onto $H_{A}$ running from the top-right of a segment to the base of the segment, and then along an edge of $H_{A}$ (Figure 17). Similarly for the bottom-left.

In Figure 17, we have drawn the tentacle by removing a bit of edge of $H_{A}$. When we do this for each segment, top-right and bottom-left, the remaining connected components of $H_{A}$ correspond exactly to ideal vertices.

Each ideal vertex of the upper polyhedron begins at the top-right (bottom-left) of a segment, and continues along a state circle of $s_{A}$ until it meets another segment. In the diagram, this will correspond to an undercrossing. As we observed above, edges terminate at undercrossings. Figure 18 shows the portions of ideal edges sketched schematically onto $H_{A}$.

As for the shaded faces, we have seen that they extend in tentacles through segments. Where do they begin? In fact, each shaded face originates from an innermost disk.

To complete our combinatorial description of the upper polyhedron, we color each innermost disk a unique color. Starting with the segments leading out of innermost disks, 

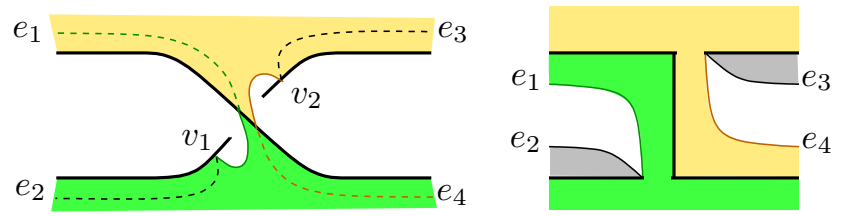

FiguRE 18. The ideal edges and shaded faces around the crossing of Figure 16
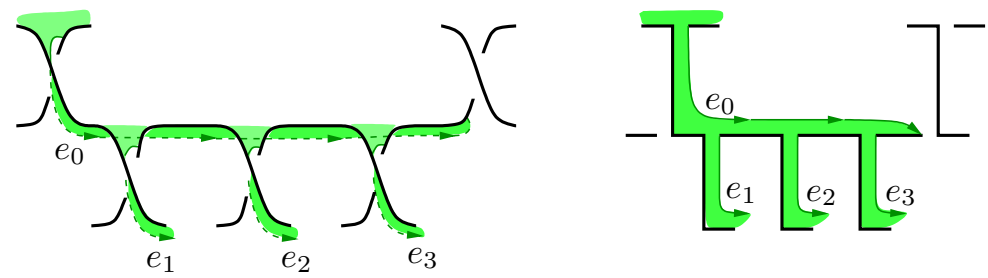

FiguRE 19. Left: part of a shaded face in an upper 3-ball. Right: the corresponding picture, superimposed on $H_{A}$. The tentacle next to the ideal edge $e_{0}$ terminates at a segment on the same side of the state circle on $H_{A}$. It runs past segments on the opposite side of the state circle, spawning new tentacles associated to ideal edges $e_{1}, e_{2}, e_{3}$.

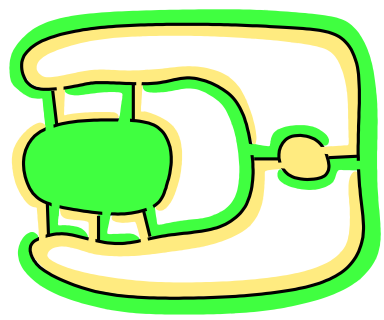

Figure 20. An example of the combinatorics of the upper polyhedron. In this example, the polyhedron is combinatorially a prism over an ideal heptagon. This prism is an $I$-bundle over a subsurface of $S_{A}$.

we sketch in tentacles, removing portions of $H_{A}$. Note that a tentacle will continue past segments of $H_{A}$ on the opposite side of the state circle without terminating, spawning new tentacles, but will terminate at a segment on the same side of the state surface. This is shown in Figure 19, Since $H_{A}$ is a finite graph, the process terminates.

An example is shown in Figure 20, For this example, there are two innermost disks, which we color green and gold. Corresponding tentacles are shown.

5.3. Prime polyhedra detect fibers. In the last subsection, we saw that the lower polyhedra correspond to alternating link diagrams. It may happen that one of these alternating diagrams is not prime. In other words, the alternating link diagram contains 
a pair of regions that meet along more than one edge. The polyhedral analogue of this notion is the following.

Definition 5.2. A combinatorial polyhedron $P$ is called prime if every pair of faces of $P$ meet along at most one edge.

Primeness is an extremely desirable property in a polyhedral decomposition, for the following reason. The theory of normal surfaces, developed by Haken [22, states that given a polyhedral decomposition of a manifold $M$, every essential surface $S \subset M$ can be moved into a form where it intersects each polyhedron in standard disks, called normal disks. If this essential surface is (say) a compression disk $D \subset M_{A}$, then we may intersect $D$ with the union of white faces $\mathcal{W}$ to form a collection of $\operatorname{arcs}$ in $D$. An outermost arc must cut off a bigon. But, by Definition 5.2 , a prime polyhedron cannot contain a bigon. Thus, once we obtain a decomposition of $M_{A}$ into prime polyhedra, Theorem 2.4 will immediately follow.

In practice, the polyhedra described in the last subsection may sometimes fail to be prime. Whenever this occurs, we need to cut them into smaller, prime pieces. Before we describe this cutting process, we record another powerful feature of the polyhedral decomposition.

Notice that the polyhedron in Figure 20 is combinatorially a prism over an ideal heptagon: here, the two shaded faces are the horizontal faces of the prism, while the seven white faces are lateral, vertical essential product disks. In other words, the entire polyhedron is an $I$-bundle over an ideal polygon. It turns out that under the hypothesis of primeness, when the upper polyhedron has this product structure, then so do all of the lower polyhedra, and so does the manifold $M_{A}=S^{3} \backslash \backslash S_{A}$.

Proposition 5.3 (Lemma 5.8 of [19]). Suppose that in the polyhedral decomposition of $M_{A}$ corresponding to an A-adequate diagram, every ideal polyhedron is prime. Then, the following are equivalent:

(1) Every white face is an ideal bigon, i.e. an essential product disk.

(2) The upper polyhedron is a prism over an ideal polygon.

(3) Every polyhedron is a prism over an ideal polygon.

(4) Every region in $R$ that corresponds to a lower polyhedron is bounded by two state circles, connected by edges of $\mathbb{G}_{A}$ that are identified to a single edge of $\mathbb{G}_{A}^{\prime}$.

(5) Every edge of $\mathbb{G}_{A}^{\prime}$ is separating, i.e. $\mathbb{G}_{A}^{\prime}$ is a tree.

Remark 5.4. In the decomposition process of $M_{A}$ that we have described so far, the polyhedra may not be prime. However, we will see in the next subsection that primeness can always be achieved after additional cutting.

The proof of equivalence of the conditions in Proposition 5.3 is not hard. For example, if every white face is a bigon, then in every polyhedron, those bigons must be strung end to end, forming a cycle of lateral faces in a prism. Note as well that if every polyhedron is a prism, i.e. a product, then this product structure extends over the bigon faces to imply that $M_{A} \cong S_{A} \times I$. This immediately gives one implication in Theorem [3.2, if $\mathbb{G}_{A}^{\prime}$ is a tree, then $S_{A}$ is a fiber. 


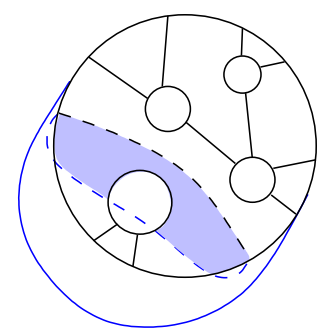

Figure 21. Split lower polyhedron along a non-prime arc.

The converse implication (if $S_{A}$ is a fiber, then $\mathbb{G}_{A}^{\prime}$ is a tree) requires knowing that our polyhedra detect the JSJ decomposition of $M_{A}$. In other words: if part (or all) of $M_{A}$ is an $I$-bundle, then this $I$-bundle structure must be visible in the individual polyhedra. This property also follows from primeness.

5.4. Ensuring Primeness. We have seen that primeness is a desirable property of the polyhedral decomposition. Here, we describe a way to detect when a lower polyhedron is not prime, and a way to fix this situation.

Definition 5.5. The graph $H_{A}$ is non-prime if there exists a state circle $C$ of $s_{A}$ and an arc $\alpha$ with both endpoints on $C$, such that the interior of $\alpha$ is disjoint from $H_{A}$, and $\alpha$ is not isotopic into $C$ in the complement of $H_{A}$. The arc $\alpha$ is called a non-prime arc.

Each non-prime arc is lies in a single white face of $\mathcal{W}$, while its shadow on the soup can below lies in a single shaded face. Thus every non-prime arc indicates that a lower polyhedron violates Definition 5.2, See Figure 21,

Whenever we find a non-prime arc, we modify the polyhedral decomposition as follows: push the arc $\alpha$ down against the soup can of the state circle $C$. This divides the corresponding lower polyhedron into two. Figure 21 shows a 3 -dimensional view of this cutting process.

Combinatorially, we cut a lower polyhedron along the non-prime arc, as in Figure 22. The lower polyhedra now correspond to alternating links whose state circles contain $\alpha$. On the boundary of the upper polyhedron, $\alpha$ meets two tentacles. These will be joined into the same shaded face, by attaching both tentacles to a regular neighborhood of $\alpha$. See Figure 23 .

Repeat this process of splitting along non-prime arcs until there are no more non-prime arcs. This ensures that all the lower polyhedra are prime. Then, one can show that the upper polyhedron is prime as well.

A decomposition along a maximal collection of non-prime $\operatorname{arcs} \mathbb{A}$ is called a prime decomposition. Its main features are summarized as follows:

- It decomposes $M_{A}$ into one upper and at least one lower polyhedron.

- Every polyhedron is prime.

- Every polyhedron is checkerboard colored, with 4-valent vertices.

- White faces of the polyhedra correspond to regions of the complement of $H_{A} \cup \mathbb{A}$.

- Lower polyhedra are in one-to-one correspondence with nontrivial complementary regions of $s_{A} \cup \mathbb{A}$. 


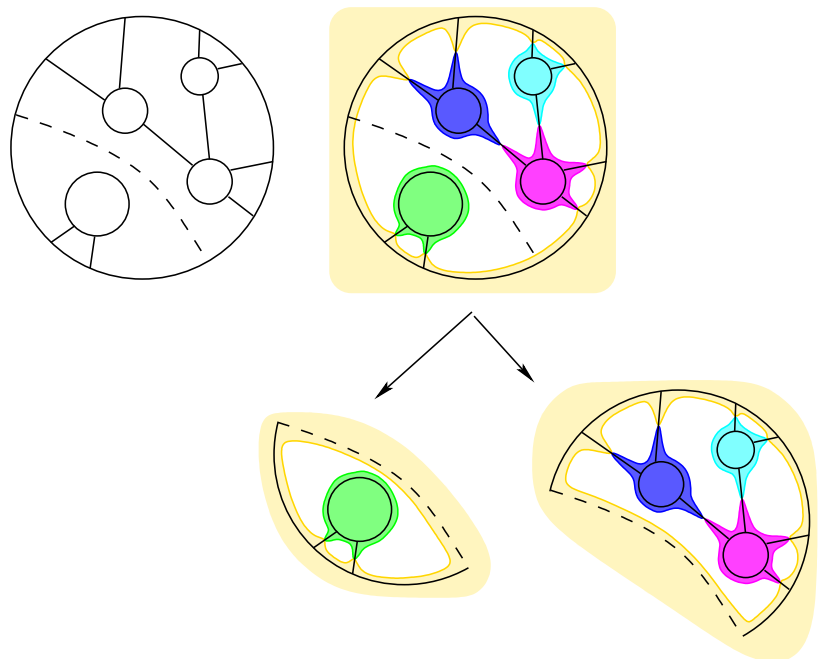

FIGURE 22. Splitting a lower polyhedron into two along a non-prime arc.

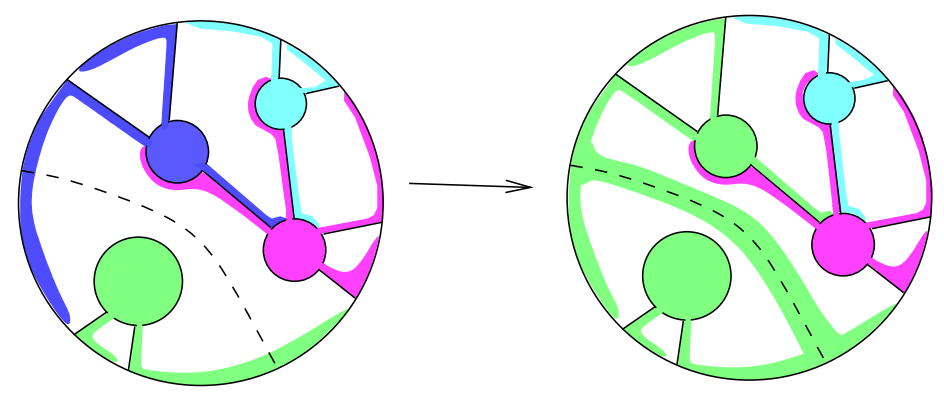

FIGURE 23. Splitting the upper polyhedron along a non-prime arc.

- Each lower polyhedron is identical to the checkerboard polyhedron of an alternating link, where the alternating link is obtained by taking the restriction of $H_{A} \cup \mathbb{A}$ to the corresponding region of $s_{A} \cup \mathbb{A}$, and replacing segments of $H_{A}$ with crossings (using the $A$-resolution).

Another important feature is that Proposition 5.3 holds for the prime polyhedral decomposition that we have just described. It is worth noting that since the lower polyhedra are are in one-to-one correspondence with nontrivial complementary regions of $s_{A} \cup \mathbb{A}$, part (4) of the proposition will refer to these regions of the diagram.

Having obtained a prime polyhedral decomposition, we may apply normal surface theory to study various pieces in the JSJ decomposition of $M_{A}$. Recall that the JSJ decomposition yields three kinds of pieces: $I$-bundles, solid tori, and the guts. To compute $\chi_{-}\left(\operatorname{guts}\left(M_{A}\right)\right)$, we need to understand the $I$-bundle components that have nonzero Euler characteristic. We prove the following.

Theorem 5.6 (Theorem 4.4 of [19]). Let B be an I-bundle component of the JSJ decomposition of $M_{A}$, such that $\chi(B)<0$. Then $B$ is spanned by a collection of essential product 
disks $\left\{D_{1}, \ldots, D_{n}\right\}$, with the property that each $D_{i}$ is embedded in a single polyhedron in the polyhedral decomposition of $M_{A}$.

The EPDs in the spanning set that lie in the lower polyhedra of the decompositions are well understood; they are in one-to-one correspondence with 2-edge loops in the state graph $\mathbb{G}_{A}$. The EPDs in the spanning set that lie in the upper polyhedron are complex; they are not obtainable in terms in the lower polyhedra. These are exactly the EPDs counted by the quantity $\left\|E_{c}\right\|$ of Theorems [3.5, 3.6 and 3.14. The EPDs in the upper polyhedron also correspond to 2-edge loops in $\mathbb{G}_{A}$, but the correspondence is not one-toone. In special cases of link diagrams, we can understand the combinatorial structure of the polyhedral decomposition well enough to show that $\left\|E_{c}\right\|=0$. This gives, for instance, Corollaries 3.15 and 3.16 .

Our results about normal surfaces in the polyhedral decomposition of $M_{A}$ can likely be used to attack other topological problems about $A$-adequate links. We refer the reader to [19. Chapter 10] for a detailed discussion of some of these open questions.

\section{REFERENCES}

[1] Colin C. Adams, Noncompact Fuchsian and quasi-Fuchsian surfaces in hyperbolic 3-manifolds, Alebr. Geom. Topol. 7 (2007), 565-582.

[2] Ian Agol, Peter A. Storm, and William P. Thurston, Lower bounds on volumes of hyperbolic Haken 3-manifolds, J. Amer. Math. Soc. 20 (2007), no. 4, 1053-1077, with an appendix by Nathan Dunfield.

[3] Cody Armond and Oliver T. Dasbach, Rogers-Ramanujan type identities and the head and tail of the colored Jones polynomial, arXiv:1106.3948.

[4] Béla Bollobás and Oliver Riordan, A polynomial invariant of graphs on orientable surfaces, Proc. London Math. Soc. (3) 83 (2001), no. 3, 513-531.

[5] _ A polynomial of graphs on surfaces, Math. Ann. 323 (2002), no. 1, 81-96.

[6] Abhijit Champanerkar, Ilya Kofman, and Eric Patterson, The next simplest hyperbolic knots, J. Knot Theory Ramifications 13 (2004), no. 7, 965-987.

[7] Marc Culler and Peter B. Shalen, Volumes of hyperbolic Haken manifolds. I, Invent. Math. 118 (1994), no. 2, 285-329.

[8] Oliver T. Dasbach, David Futer, Efstratia Kalfagianni, Xiao-Song Lin, and Neal W. Stoltzfus, The Jones polynomial and graphs on surfaces, Journal of Combinatorial Theory Ser. B 98 (2008), no. 2, 384-399.

[9] _ Alternating sum formulae for the determinant and other link invariants, J. Knot Theory Ramifications 19 (2010), no. 6, 765-782.

[10] Oliver T. Dasbach and Xiao-Song Lin, On the head and the tail of the colored Jones polynomial, Compositio Math. 142 (2006), no. 5, 1332-1342.

[11] _ A volume-ish theorem for the Jones polynomial of alternating knots, Pacific J. Math. 231 (2007), no. 2, 279-291.

[12] Tudor Dimofte and Sergei Gukov, Quantum field theory and the volume conjecture, Contemp. Math. 541 (2011), 41-68.

[13] Nathan M. Dunfield and Stavros Garoufalidis, Incompressibility criteria for spun-normal surfaces, Trans. Amer. Math. Soc. 364 (2012), no. 11, 6109-6137.

[14] David Futer, Efstratia Kalfagianni, and Jessica S. Purcell, Dehn filling, volume, and the Jones polynomial, J. Differential Geom. 78 (2008), no. 3, 429-464.

[15] - Symmetric links and Conway sums: volume and Jones polynomial, Math. Res. Lett. 16 (2009), no. 2, 233-253.

[16] Cusp areas of Farey manifolds and applications to knot theory, Int. Math. Res. Not. IMRN 2010 (2010), no. 23, 4434-4497. 
[17] - On diagrammatic bounds of knot volumes and spectral invariants, Geom. Dedicata 147 (2010), $115-130$.

[18] Slopes and colored Jones polynomials of adequate knots, Proc. Amer. Math. Soc. 139 (2011), $1889-1896$.

[19] - Guts of surfaces and the colored Jones polynomial, Research monograph to appear in Lecture Notes in Mathematics (Springer), vol. 2069, arXiv:1108.3370.

[20] Stavros Garoufalidis, The Jones slopes of a knot, Quantum Topol. 2 (2011), no. 1, 43-69.

[21] Stavros Garoufalidis and Thang T. Q. Lê, The colored Jones function is q-holonomic, Geom. Topol. 9 (2005), 1253-1293 (electronic).

[22] Wolfgang Haken, Theorie der Normalflächen, Acta Math. 105 (1961), 245-375.

[23] Allen E. Hatcher, On the boundary curves of incompressible surfaces, Pacific J. Math. 99 (1982), no. 2, $373-377$.

[24] Jim Hoste and Morwen B. Thistlethwaite, Knotscape, http://www.math.utk.edu/〜morwen.

[25] William H. Jaco and Peter B. Shalen, Seifert fibered spaces in 3-manifolds, Mem. Amer. Math. Soc. 21 (1979), no. 220, viii+192.

[26] Klaus Johannson, Homotopy equivalences of 3-manifolds with boundaries, Lecture Notes in Mathematics, vol. 761, Springer, Berlin, 1979.

[27] Vaughan F. R. Jones, Hecke algebra representations of braid groups and link polynomials, Ann. of Math. (2) 126 (1987), no. 2, 335-388.

[28] Rinat M. Kashaev, The hyperbolic volume of knots from the quantum dilogarithm, Lett. Math. Phys. 39 (1997), no. 3, 269-275.

[29] Marc Lackenby, The volume of hyperbolic alternating link complements, Proc. London Math. Soc. (3) 88 (2004), no. 1, 204-224, With an appendix by Ian Agol and Dylan Thurston.

[30] W. B. Raymond Lickorish, An introduction to knot theory, Graduate Texts in Mathematics, vol. 175, Springer-Verlag, New York, 1997.

[31] W. B. Raymond Lickorish and Morwen B. Thistlethwaite, Some links with nontrivial polynomials and their crossing-numbers, Comment. Math. Helv. 63 (1988), no. 4, 527-539.

[32] William W. Menasco, Polyhedra representation of link complements, Low-dimensional topology (San Francisco, Calif., 1981), Contemp. Math., vol. 20, Amer. Math. Soc., Providence, RI, 1983, pp. 305325.

[33] — Closed incompressible surfaces in alternating knot and link complements, Topology 23 (1984), no. 1, 37-44.

[34] Yosuke Miyamoto, Volumes of hyperbolic manifolds with geodesic boundary, Topology 33 (1994), no. 4, 613-629.

[35] Hitoshi Murakami, An introduction to the volume conjecture, Contemp. Math. 541 (2011), 1-40.

[36] Hitoshi Murakami and Jun Murakami, The colored Jones polynomials and the simplicial volume of a knot, Acta Math. 186 (2001), no. 1, 85-104.

[37] Makoto Ozawa, Essential state surfaces for knots and links, J. Aust. Math. Soc. 91 (2011), no. 3, 391-404.

[38] Józef H. Przytycki, From Goeritz matrices to quasi-alternating links, The mathematics of knots, Contrib. Math. Comput. Sci., vol. 1, Springer, Heidelberg, 2011, pp. 257-316.

[39] Jessica S. Purcell, Volumes of highly twisted knots and links, Algebr. Geom. Topol. 7 (2007), 93-108.

[40] Alexander Stoimenow, On the crossing number of semi-adequate links, Preprint available at http:// stoimenov.net/stoimeno/homepage/papers/adeq2.pdf.

[41] Morwen B. Thistlethwaite, On the Kauffman polynomial of an adequate link, Invent. Math. 93 (1988), no. 2, 285-296.

[42] William P. Thurston, Three-dimensional manifolds, Kleinian groups and hyperbolic geometry, Bull. Amer. Math. Soc. (N.S.) 6 (1982), no. 3, 357-381.

[43] — A norm for the homology of 3-manifolds, Mem. Amer. Math. Soc. 59 (1986), no. 339, i-vi and $99-130$.

[44] Vladimir G. Turaev, A simple proof of the Murasugi and Kauffman theorems on alternating links, Enseign. Math. (2) 33 (1987), no. 3-4, 203-225. 
JONES POLYNOMIALS, VOLUME, AND ESSENTIAL KNOT SURFACES: A SURVEY

[45] - The Yang-Baxter equation and invariants of links, Invent. Math. 92 (1988), no. 3, 527-553.

[46] Edward Witten, 2+1-dimensional gravity as an exactly soluble system, Nuclear Phys. B 311 (1988/89), no. $1,46-78$.

[47] _ Quantum field theory and the Jones polynomial, Comm. Math. Phys. 121 (1989), no. 3, 351-399.

Department of Mathematics, Temple University, Philadelphia, PA 19122, USA

E-mail address: dfuter@temple.edu

Department of Mathematics, Michigan State University, East Lansing, Mi 48824, USA

E-mail address: kalfagia@math.msu.edu

Department of Mathematics, Brigham Young University, Provo, UT 84602, USA

E-mail address: jpurcell@math.byu.edu 